\title{
NOTE ON QUASI-DECOMPOSITIONS OF IRREDUCIBLE GROUPS ${ }^{1}$
}

\author{
E. F. CORNELIUS, JR.
}

\begin{abstract}
A quasi-decomposition theorem is obtained for a torsion free abelian group with quasi-endomorphism algebra satisfying the minimum condition on left ideals.
\end{abstract}

Several quasi-decomposition theorems of J. D. Reid and R. S. Pierce for torsion free abelian groups of finite rank can be extended to groups of arbitrary rank by replacing the finite rank hypothesis with the requirement that the quasi-endomorphism algebra of the groups satisfy the minimum condition on left ideals. This minimum condition can also be characterized topologically. The lemma below is the key to these generalizations; that they are nontrivial is illustrated by familiar examples such as groups of $p$-adic integers.

Hereafter the term "group" refers to a reduced, torsion free abelian group. The discussion is normalized by considering only subgroups of a fixed vector space $V$ over the rational number field $Q$. The algebra of linear transformations of $V, L(V)$, is equipped with the finite topology [4]. Basic results about quasi-isomorphism are assumed; for a complete background consult [1], [6], [7]. $\doteq, \doteq, \dot{\sim}$ denote quasi-contained, quasi-equal, and quasi-isomorphic, respectively. $G$ will always denote a full subgroup of $V$, i.e., a subgroup with torsion quotient, $V / G$. Recall that $Q E(G)=\{f \in L(V): f G \subseteq G\}$ is the quasiendomorphism algebra of $G$. $H^{*}$ denotes the rational subspace of $V$ spanned by a subgroup $H$ of $V$. Finally, all sums are direct.

After Reid [7], call $G$ irreducible if and only if it has no nontrivial pure, fully invariant subgroups. It is easy to see that an irreducible group is homogeneous; thus we lose no generality by considering only reduced groups. Reid shows that $G$ is irreducible if and only if $V$ is an irreducible $Q E(G)$-module by establishing a one-to-one correspondence between the pure, fully invariant subgroups of $G$ and the $Q E(G)$-submodules of $V$. It follows readily that a quasi-summand of an irreducible group is itself irreducible and thus that irreducibility

Received by the editors January 6, 1970 .

AMS 1969 subject classifications. Primary 2030; Secondary 1625, 1698.

Key words and phrases. Quasi-isomorphism, quasi-decomposition, quasi-endomorphism algebra, minimum condition, finite topology.

1 These results appear in Chapter 1 of the author's doctoral thesis supervised by Professor R. A. Beaumont at the University of Washington, Seattle, Washington. 
is invariant under quasi-isomorphism. For irreducible $G$, the closure of $Q E(G)$ in $L(V)$ is $\operatorname{Hom}_{C(G)}(V, V)$, with $C(G)=\operatorname{Hom}_{Q E(G)}(V, V)$ $[4$, p. 31]. Our immediate goal is to extend Theorem 5.5 [7] to groups of arbitrary rank. Lemma 1.1 enables us to do this by providing the necessary finiteness condition.

LEMMA 1.1. Let $V$ be a vector space over a division algebra $D$ and suppose that $\operatorname{Hom}_{D}(V, V) \subseteq Q E(G)$. Then $V$ is finite dimensional over $D$.

Proof. Suppose $\left\{a_{i}\right\}_{i=1}^{\infty}$ is a subset of an infinite $D$-basis of $V$, contained in $G$ ( $G$ is full in $V$ ). Since $G$ is reduced, $p_{i}^{-n i} a_{i} \notin G$ for some prime $p_{i}$ and some positive integer $n_{i}, i=1, \ldots, \infty$. But no $D$-linear transformation sending basis element $a_{i}$ to $p_{i}^{-i n i} a_{i}, i=1, \ldots, \infty$, is a quasi-endomorphism of $G$. This contradiction shows that $V$ must have finite $D$-dimension.

THEOREM 1.2. These conditions on the group $G$ are equivalent.

(1) $G$ is irreducible and $Q E(G)$ satisfies the minimum condition on left ideals.

(2) $G$ is irreducible and $Q E(G)$ is closed in the finite topology of $L(V)$.

(3) $Q E(G)=\operatorname{Hom}_{D}(V, V)$ with $V$ a vector space over a division algebra $D$. Consequently $V$ is finite m-dimensional over $D=C(G)$.

(4) $G \doteq \sum_{i=1}^{m} G_{i}$ with each $G_{i} \underset{\sim}{\sim} H, H$ irreducible, and $Q E(H)$ a division algebra.

Proof. (1) implies (2). $V$ is an irreducible $Q E(G)$-module because $G$ is an irreducible group. Thus by Theorem $1[4$, p. 39] and the Density Theorem [4, p. 31], $Q E(G)$ is equal to its closure and so is closed.

(2) implies (3). $C(G)$ is a division algebra because $G$ is irreducible and $Q E(G)=\operatorname{Hom}_{C(G)}(V, V)$ by the Density Theorem [4, p. 31].

(3) implies (4). Suppose $V$ is a vector space over a division algebra $D$ and $Q E(G)=\operatorname{Hom}_{D}(V, V)$. By Theorem $1[4$, p. 32], $D=C(G)$ and by Lemma 1.1, $V$ is finite $m$-dimensional over $D$. We further observe that $G$ is irreducible because $V$ is an irreducible $Q E(G)$-module. Thus if $e_{1}, \cdots, e_{m}$ are projections in $\operatorname{Hom}_{D}(V, V)$ giving a decomposition of $V$ into one-dimensional $D$-spaces, then $G \doteq \sum_{i=1}^{m} e_{i} G$ is a quasidecomposition of $G$ into irreducible groups $e_{i} G$ such that $Q E\left(e_{i} G\right)$ $=e_{i} Q E(G) e_{i}[6]$ is a division algebra, $i=1, \cdots, m$. Finally, the $e_{i} G$ are quasi-isomorphic groups because the $Q E(G) e_{i}$ are isomorphic $Q E(G)$-modules [6].

(4) implies (1). Let $e_{1}, \cdots, e_{m}$ be idempotents corresponding to the given quasi-decomposition of $G$. The $Q E(G) e_{i}$ are isomorphic $Q E(G)$-modules because the $G_{i}$ are quasi-isomorphic groups [6]. Thus 
$\left[4\right.$, p. 52], $e_{1}, \cdots, e_{m}$ are diagonal matrix units in $Q E(G)$. Since $e_{1} Q E(G) e_{1}=Q E\left(G_{1}\right) \cong Q E(G)$ [6] is a division algebra, $Q E(G)$ is isomorphic to the ring of $m \times m$ matrices over a division algebra and thus satisfies the minimum condition. It is easy to see that $V$ is an irreducible $Q E(G)$-module and so $G$ is irreducible.

REMARK 1.3. In Theorem $1.2, m$ is the unique number of quasisummands in every quasi-decomposition of $G$ into strongly indecomposable groups [6].

COROllary 1.4. Let $G$ be both irreducible and strongly indecomposable. Then if $Q E(G)$ satisfies the minimum condition, it is a division algebra.

Corollary 1.5. $Q E(G)=L(V)$ if and only if $G$ is the direct sum of a finite number of isomorphic rank-one subgroups.

Proof. If $G=\sum_{i=1}^{m} G_{i}$ with the $G_{i}$ isomorphic rank-one subgroups, then $G$ satisfies (4) of Theorem 1.2. Thus in (3), $D=Q$ because $m=$ rank $G=[V: Q]=[V: D][D: Q]=m[D: Q]$, where the brackets denote vector space dimension over the indicated field. Conversely, suppose $Q E(G)=L(V)$; then (3) of Theorem 1.2 holds. Now $D=Q$ and so $m=\operatorname{rank} G$; by (4), $G \doteq \sum_{i=1}^{m} G_{i}$ with the $G_{i}$ quasi-isomorphic rank-one groups. Corollary 9.2 [1] and Corollary $9.6[1]$ complete the proof.

By judiciously employing Lemma 1.1 and arguments similar to the above, results of Pierce [5] can be extended to full subrings of simple rational algebras of arbitrary dimension over $Q$ [2]. Reid [7] has shown that the additive group of such subrings is irreducible.

We conclude with several examples. It is well known [3, p. 212] that every endomorphism of the $p$-adic integers $J_{p}$ is just multiplication by some $p$-adic integer. It follows that $Q E\left(J_{p}\right)=F_{p}$, the $p$-adic number field. By Theorem 1.2, any finite direct sum of $J_{p}$ is a group (of uncountable rank) with quasi-endomorphism algebra satisfying the minimum condition on left ideals. Likewise, if $(R)_{m}$ denotes the ring of all $m \times m$ matrices over the ring $R$, then $\left(J_{p}\right)_{m}$ as a subring of $\left(F_{p}\right)_{m}$ has a field of definition [5] in a generalized sense [2].

\section{BiBLIOGRAPHY}

1. R. A. Beaumont and R. S. Pierce, Torsion-free rings, Illinois J. Math. 5 (1961), 61-98. MR $26 \# 6212$.

2, E. F. Cornelius, Jr., The quasi-endomorphism algebra of a torsion free abelian group, Doctoral Thesis, University of Washington, Seattle, Wash., 1969, pp. 1-13. 
3. L. Fuchs, Abelian groups, Akad. Kiad6, Budapest, 1958; reprint, Internat. Series of Monographs of Pure and Appl. Math., Pergamon Press, New York, 1960. MR 21 \#5672; MR 22 \#2644.

4. N. Jacobson, Structure of rings, Amer. Math. Soc. Colloq. Publ., vol. 37, Amer. Math. Soc., Providence, R.I., 1956. MR 18, 373.

5. R. S. Pierce, Subrings of simple algebras, Michigan Math. J. 7 (1960), 241-243. MR $26 \# 6213$.

6. J. D. Reid, On quasi-decomposition of torsion free Abelian groups, Proc. Amer. Math. Soc. 13 (1962), 550-554. MR 25 \#1209.

7. - On the ring of quasi-endomorphisms of a torsion-free group, Proc. Sympos. Topics in Abelian Groups (New Mexico State Univ., 1962), Scott, Foresman, Chicago, Ill., 1963, pp. 51-68. MR 30 \#158.

Wayne State University, Detroit, Michigan 48202 